\title{
Interleukin-6 receptor blockade induces limited repair of bone erosions in rheumatoid arthritis: a micro CT study
}

\author{
Stephanie Finzel, ${ }^{1}$ Juergen Rech, ${ }^{1}$ Sarah Schmidt, ${ }^{1}$ Klaus Engelke, ${ }^{2}$ \\ Matthias Englbrecht, ${ }^{1}$ Georg Schett ${ }^{1}$
}

${ }^{1}$ Department of Internal Medicine 3, University of Erlangen-Nuremberg, Erlangen, Germany

2Institute of Medical Physics, University of Erlangen-

Nuremberg; Erlangen, Erlangen, Germany

\section{Correspondence to}

Georg Schett, Department of Internal Medicine

3, Rheumatology and Immunology, University of Erlangen-Nuremberg; Krankenhausstrasse 12 , Erlangen D-91054; Germany; georg.schett@uk-erlangen.de

Accepted 9 April 2012 Published Online First 14 May 2012

\begin{abstract}
Introduction Interleukin-6 receptor (IL-6R) blockade improves the signs and symptoms of rheumatoid arthritis (RA) and retards bone damage. Whether IL-6R blockade allows repair of existing bone erosions is so far unclear. Methods This study examined bone erosions in the metacarpophalangeal joints of 20 patients receiving treatment with the IL-6R blocker tocilizumab using micro CT ( $\mu \mathrm{CT})$. The maximal width and depth of individual bone erosions was measured at baseline and after 1 year of treatment.

Results 133 bone erosions were identified at baseline with a mean $( \pm S D)$ size of $2.23 \pm 1.26 \mathrm{~mm}$ and depth of $2.16 \pm 1.50 \mathrm{~mm}$. Distribution analysis showed predominant involvement of the second compared with the third and fourth metacarpophalangeal joints, the metacarpal heads compared with the phalangeal bases and the radial quadrants compared with all other surfaces. Repair of bone erosions during tocilizumab treatment was confined to those lesions showing sclerosis at baseline and/or at follow-up and those with a width larger than $1.6 \mathrm{~mm}$. The mean decrease in width of sclerosed erosions was thus $0.14 \pm 0.05 \mathrm{~mm}(p=0.0086)$ and $0.20 \pm 0.08 \mathrm{~mm}(p=0.019)$ for sclerosing lesions after 1 year of treatment.

Conclusions Blockade of IL-6R by tocilizumab can induce limited repair in a subset of erosions, particularly in large lesions with sclerosis. Repair of erosions during tocilizumab treatment reflects the favourable impact of IL-6R blockade on local bone remodelling in patients with RA.
\end{abstract}

Bone erosion is a hallmark of rheumatoid arthritis (RA) reflecting the tight interaction between inflammation, structural damage and functional disability. ${ }^{1-3}$ Bone erosion results from a chronic inflammatory process in the synovial membrane and adjacent bone marrow, which triggers skeletal damage particularly at the 'bare areas' of the joint, where bone is not covered by cartilage. Pathogenesis of bone erosion is based on local expression of inflammatory cytokines, such as the receptor activator of nuclear factor kappa B ligand (RANKL) and tumour necrosis factor (TNF) alpha, which stimulate the differentiation of synovial monocytes/macrophages into osteoclasts and triggers the resorption of cortical bone. ${ }^{4}$ Destruction of bone and the covering cartilage has been shown to be closely related to functional decline in RA patients, ${ }^{23}$ and antirheumatic therapies attempt to retard, stop or even reverse structural bone damage in arthritis to maintain or even improve the patients' disease state. ${ }^{5}$

Antirheumatic drugs show specific effects on the periarticular bone, which are related to the magnitude and the quality of their bone-protective action. ${ }^{5}$ Bone-protective effects of antirheumatic drugs are usually based on their anti-inflammatory properties, which allow control of synovitis, and their direct effects on the bone-resorptive process by affecting osteoclast differentiation. Retardation and even arrest of progression of bone erosion is a well-known effect of antirheumatic drugs, which appears to be particularly strong with TNF blocking agents. Much less, however, is known about the possibility of repair of existing bone erosions and whether and how cytokine blockade influences this process.

The principal possibility of repair of existing bone erosions has been obtained from radiographic studies, ${ }^{6-9}$ which have been later supported by MRI, CT and ultrasound. ${ }^{10} 11$ We have recently developed a high-resolution imaging technique to measure the dimensions of single bone erosions exactly. ${ }^{12}$ Its high resolution combined with direct visualisation of bone allows micro CT ( $\mu \mathrm{CT})$ to detect even tiny changes in the dimensions of single bone erosions and thereby allows the assessment of repair. By using $\mu \mathrm{CT}$, it has been shown that repair is associated with the apposition of bone at the bottom of the lesions resulting in partial refilling of the lesions. ${ }^{13}$

It is unknown whether other cytokine-blocking strategies also allow repair of existing bone erosions. We therefore decided to search for evidence of repair in RA patients treated with the neutralising interleukin-6 receptor (IL-6R) antibody tocilizumab, because interleukin 6 (IL-6) has been shown to regulate bone homeostasis by affecting osteoblast and osteoclast function. ${ }^{14}$ To accomplish this goal, we performed a sequential $\mu \mathrm{CT}$ analysis of bone erosions in RA patients treated with tocilizumab over 1 year to determine exactly the occurrence of repair.

\section{METHODS}

\section{Patients}

Twenty RA patients were included in this study. All patients fulfilled the 1987 American Rheumatism Association revised criteria for the classification of 
RA, ${ }^{15}$ as well as the new 2010 American College of Rheumatology/ European League Against Rheumatism classification criteria for $\mathrm{RA},{ }^{16}$ and were recruited at the rheumatology outpatient clinic of the University Clinic of Erlangen. All patients received treatment with the neutralising antibody against IL-6R tocilizumab at a dose of $8 \mathrm{mg} / \mathrm{kg}$ body weight in combination with methotrexate (mean dose of methotrexate $12.88 \mathrm{mg} /$ week). Treatment of tocilizumab as well as methotrexate was stable for at least 3 months before the baseline $\mu \mathrm{CT}$ examination. The study was performed in accordance with the Declaration of Helsinki. Approval from the local ethics committee and national radiation safety agency (Bundesamt für Strahlenschutz) as well as written informed consent was obtained for the study.

\section{Imaging procedures}

All patients received a $\mu$ CT scan of the second to fourth metacarpophalangeal joints of the right hand at baseline (start of tocilizumab treatment) and at follow-up after 1 year. The $\mu \mathrm{CT}$ scans were performed with an XtremeCT scanner (SCANCO Medical AG, Brüttisellen, Switzerland) at a resolution of $82 \times 82 \times 82 \mu \mathrm{m}$ voxel size. The scan region was 80 slices distal and 242 slices proximal of the upper margin of the third metacarpal head. Scan time was 8 min per patient and scan. For exact positioning the hand was positioned in a neutral posture and was padded. ${ }^{10} \mathrm{~A}$ single investigator (SF) trained in the technique performed all scans.

\section{Evaluation of images}

The width and depth of bone erosions in the metacarpophalangeal joint of the second, third and fourth fingers were assessed. Erosions were defined as a break of the juxta-articular cortical bone. All erosions were documented both at baseline and after 1 year (follow-up). Evaluation of overall 322 two-dimensional $\mu \mathrm{CT}$ slices in the axial, sagittal and coronal plane was performed using the open source DICOM viewer (Rosslyn, VA, USA) Osirix V3.2. Assessment included the palmar, ulnar, dorsal as well as the radial quadrants of the metacarpal heads and the phalangeal bases. ${ }^{12}$ Individual bone erosions were both characterised by metrical assessment of the maximal width and depth of the cortical breaks. ${ }^{12}$ In addition, any signs of bone apposition at the base of the erosion were documented. Evaluation of the maximal width of erosion, maximal depth of erosion and the presence/absence of sclerosis was performed by two readers (KE and SS), blinded to the sequence of scans as well as the patient's identity. Based on the presence or absence of sclerosis at baseline or follow-up, respectively, erosions were classified as 'non-sclerosing', in which all erosions showed no bone apposition either at baseline or at follow-up; 'sclerosing' erosions were defined as bone apposition absent at baseline but present at follow-up; 'sclerosed' were all erosions with bone apposition both at baseline and follow-up, as described previously. ${ }^{13}$ Intraobserver reproducibility both for erosion width and depth (SS) was very high (intraclass correlation 0.99) and also interobserver reproducibility (KE and SS) was high (intraclass correlations: erosion width 0.93 , erosion depth 0.94 ). The smallest detectable change of erosion depth and erosion width was calculated on the basis of 25 individual erosions, as suggested by Bruynesteyn et $\mathrm{al}^{17}$ and was extremely low $(0.008 \mathrm{~mm}$ both for erosion width and depth). Readings performed by SS were used for further comparisons.

\section{Statistics}

The width and depth of cortical breaks were Gaussian distributed at baseline and at follow-up. Paired student's t test was used for comparison of groups. Calculations were performed with SPSS program version 17.0. A p value of less than 0.05 was considered statistically significant.

\section{RESULTS}

\section{Baseline characteristics of patients and bone erosions}

Twenty patients receiving tocilizumab therapy were included in this study: all of them were followed over 1 year and received $\mu$ CT-based assessment of the second, third and fourth metacarpophalangeal joints to measure exactly all bone erosions at baseline after 1 year for reassessment. Seventeen patients were women and three were men, with a mean \pm SD age of $50.7 \pm 13.5$ years and a mean \pm SD disease duration of $11.1 \pm 8.8$ years. Mean disease activity at baseline according to the disease activity score using 28 joint counts (DAS28) was $5.48 \pm 2.38$, at baseline showing active disease with significant improvement in the signs and symptoms of RA after 1 year (mean \pm SD DAS28 3.25 \pm 1.13 ).

We found at total number of 133 bone erosions at baseline. All these 133 lesions could be visualised again at follow-up examination. In addition, nine new erosions were detected at followup. The mean $( \pm \mathrm{SD})$ width of bone erosions was $2.23 \pm 1.26 \mathrm{~mm}$, mean $( \pm \mathrm{SD})$ depth was $2.16 \pm 1.50 \mathrm{~mm}$. Erosions showed a standard distribution pattern, as reported previously, ${ }^{12}$ with preferential involvement of the second (49\%) compared with the third (29\%) and fourth (22\%) metacarpophalangeal joints, the metacarpal heads $(62 \%)$ compared with the phalangeal bases $(38 \%)$ and the radial joint compartment $(37 \%)$ compared with the ulnar (26\%), dorsal (19\%) and palmar (18\%) joint compartments (figure 1).

\section{Effects of tocilizumab therapy on the size of individual bone erosions}

We next compared the changes in maximal width and depth of the 133 bone erosions found at baseline and follow-up examination. The width of bone erosions significantly $(p=0.014)$

\section{Distribution of Erosions}

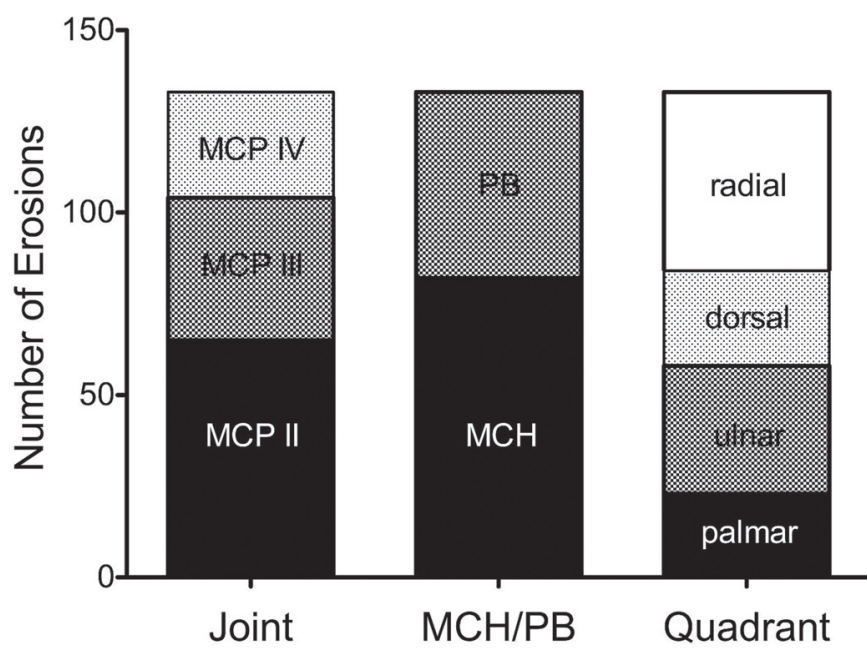

Figure 1 Distribution of bone erosions. Bar graph showing the distribution of bone erosions ( $N=133$ ) based on the type of metacarpophalangeal (MCP) joint (second, third or fourth; left), the distribution among metacarpal heads $(\mathrm{MCH})$ or the phalangeal bases (PB; phalangeal bases; middle) or the quadrant of the MCP joint (radial, ulnar, dorsal or palmar). 


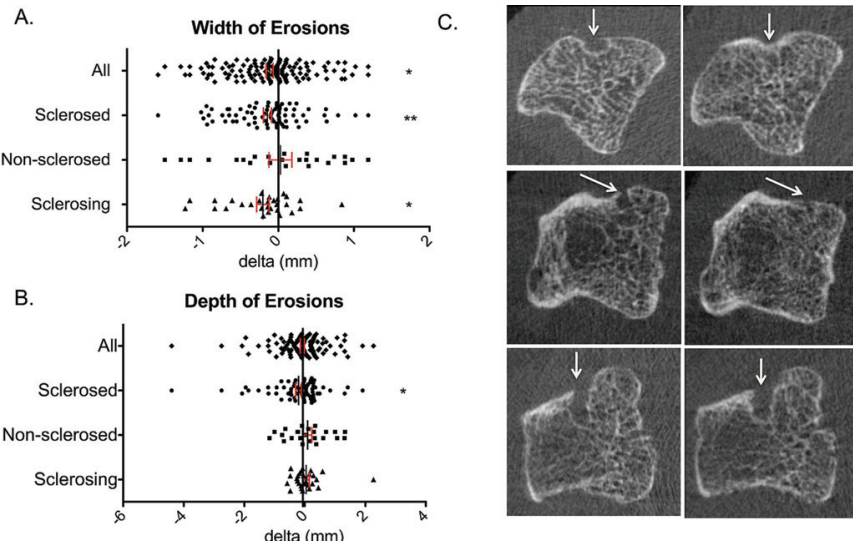

Figure 2 Evidence for repair of bon e erosions in rheumatoid arthritis patients treated with tocilizumab. (A, B) Changes in maximal width (A) and depth (B) of individual bone erosions from baseline after 1 year of tocilizumab treatment. Results are shown for all erosive lesions and subsets with signs of sclerosis at baseline and follow-up (scleroses), with sclerosis only at follow-up (sclerosing) and those with no signs of sclerosis (non-sclerosing). Red bars indicate $95 \% \mathrm{Cl}$. (C) Representative bone erosions (white arrows) at base line and follow-up. Asterisks indicate significant difference from baseline $\left({ }^{*} p<0.05,{ }^{* *} p<0.01\right)$.

decreased after 1 year of tocilizumab therapy (mean \pm SEM change from baseline $-0.11 \pm 0.04 \mathrm{~mm}$; median -0.08 ). The mean depth of bone erosions showed some decrease after 1 year attributing to a mean \pm SEM change of $-0.08 \pm 0.06$ $\mathrm{mm}$ (median -0.03), but results did not reach significance (figure 2A,B).

We have previously shown that sclerosis in individual bone erosions is associated with a reduction in the size of lesions in patients treated with TNF blocking agents. ${ }^{13}$ Indeed, the association between sclerosis and repair was also seen in patients receiving tocilizumab. Erosions with no evidence of sclerosis at baseline and follow-up ('non-sclerosing') did not show evidence of repair (mean \pm SEM change in width $0.02 \pm 0.15 \mathrm{~mm}$, mean \pm SEM change in depth $0.09 \pm 0.14 \mathrm{~mm}$ ). However, the absolute number of non-sclerosing lesions was rather low $(\mathrm{N}=24)$. In contrast, erosions showing sclerosis at baseline ('sclerosed') were most abundant $(\mathrm{N}=78)$ and showed clear evidence of repair (mean \pm SEM change in width $-0.14 \pm 0.05$ $\mathrm{mm}, \mathrm{p}=0.0086$; mean \pm SEM change in depth $-0.20 \pm 0.09 \mathrm{~mm}$, $\mathrm{p}=0.038$ ). Also, lesions with no sclerosis at baseline but sclerosis at follow-up ('sclerosing', $\mathrm{N}=31$ ) showed evidence of repair, particularly in the reduction in the width of lesions (mean \pm SEM change in width $-0.20 \pm 0.08 \mathrm{~mm}, \mathrm{p}=0.019$; mean \pm SEM change in depth $0.05 \pm 0.09 \mathrm{~mm}$, not significant) (figure 2A,B). Representative examples of bone erosion showing evidence of repair are shown in figure $2 \mathrm{C}$.

\section{Anatomical distribution of erosion repair in RA patients treated with tocilizumab}

We were next interested in whether repair preferentially occurs at certain anatomical areas. We therefore analysed the mean changes of erosion width and depth at 24 different joint regions (figure 3). The analysis of erosion width showed that those joint compartments most affected by bone erosions such as the second metacarpophalangeal joint reveal the most consistent evidence of repair. When analysing the anatomical predilection sites for the reduction in erosion depth the radial compartments of the second and third metacarpal heads showed most evidence of repair.
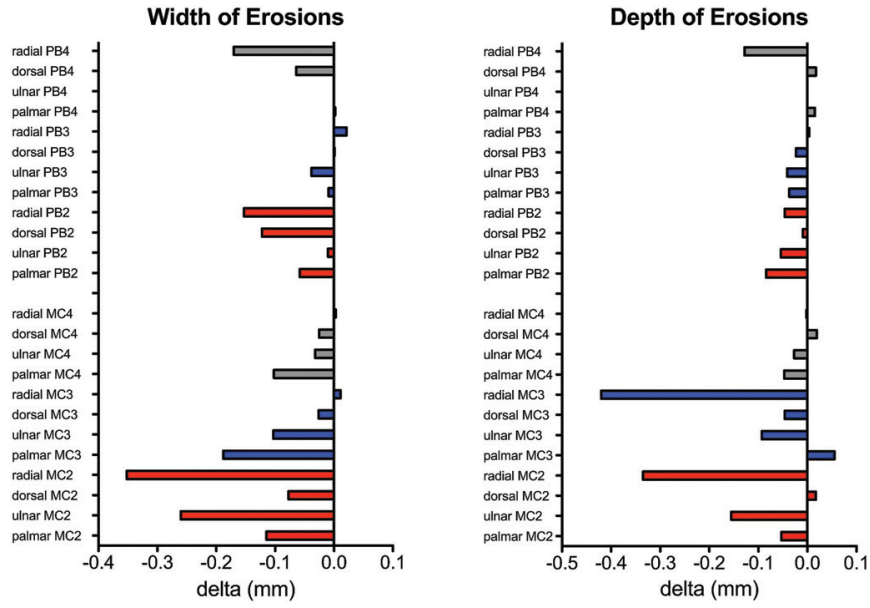

Figure 3 Anatomical distribution of repair. Changes in the width and depth of erosive lesions were documented in 24 different anatomical sub-compartments. These include 12 regions at the metacarpal (MC) heads and 12 regions at the phalangeal bases (PB). These 12 regions are based on the radial, dorsal, ulnar and palmar compartments of the second (red), third (blue) and fourth (grey) metacarpophalangeal joints. $\mathrm{X}$-axis indicates mean changes in erosions.

Erosion size and repair in RA patients treated with tocilizumab We next analysed whether the size of erosions predicts repair. We therefore created quartiles according to the width and depth of bone erosions (figure $4 \mathrm{~A}, \mathrm{~B}$ ). We performed this analysis for both sclerosed and sclerosing bone erosions, as evidence of repair was confined to these two categories of erosions. Analysis of different quartiles for erosion width showed that repair was confined to larger lesions of more than $1.76 \mathrm{~mm}$ (quartiles 3 and 4; both $\mathrm{p}<0.05$ ) for sclerosed lesions and $2.55 \mathrm{~mm}$ (quartiles 3 and 4 ; both $\mathrm{p}<0.05$ ) for sclerosing lesions. Similar results were obtained when analysing the different quartiles of erosion depth. Sclerosed lesions with a depth of more than $1.60 \mathrm{~mm}$ (quartiles 3 and 4 , both $p<0.05$ ) but not the smaller ones (quartiles 1 and 2 ) showed evidence of repair. With sclerosing lesions those over $3.31 \mathrm{~mm}$ in depth (quartile 4) showed a strong trend towards repair, although results did not reach statistical significance.

The preponderance of repair in larger bone erosions also became evident from the analysis of probability plots for changes in erosion width and depth. When plotting changes in erosion width and depth in patients treated with tocilizumab, lesions with repair, arrest and progression can be identified (figure 4C). Larger lesions (quartiles 3 and 4) accumulated in the left negative part of the probability plot indicating a higher prevalence of repair (figure 4D). For instance $71.4 \%$ of the lesions with evidence of substantial repair ( $>0.5 \mathrm{~mm}$ decrease in width) were larger lesions, whereas only $6.7 \%$ of lesions with substantial progression ( $>0.5 \mathrm{~mm}$ increase in width) were larger erosions.

\section{DISCUSSION}

By using $\mu \mathrm{CT}$, a high-resolution imaging technique designed for the quantitative analysis of bone, we show that therapy with the IL-6R blocker tocilizumab allows limited repair of bone erosions. Although repair was incomplete and all of the 133 bone erosions detected at baseline could be revisited at follow-up, treatment with tocilizumab was associated with an improvement in the width of cortical breaks as well as their depth. Repair was closely linked to evidence of sclerosis, which probably reflects new bone formation as well as the baseline size of erosions, with larger lesions showing a higher likelihood of 
A.

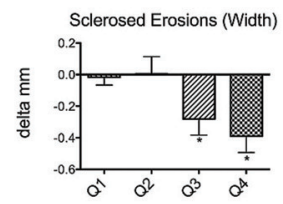

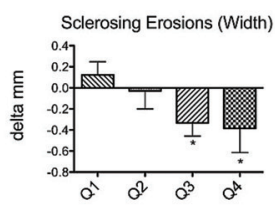

B.
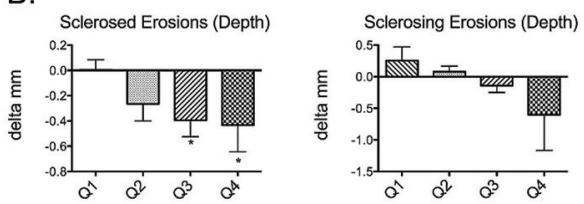

C.

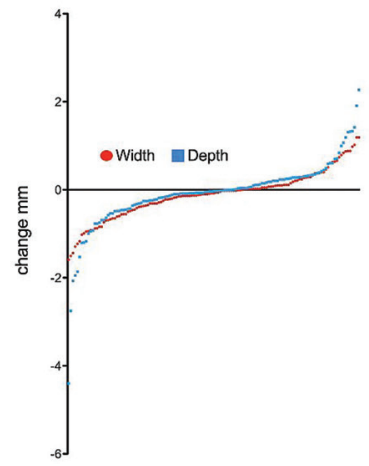

D.
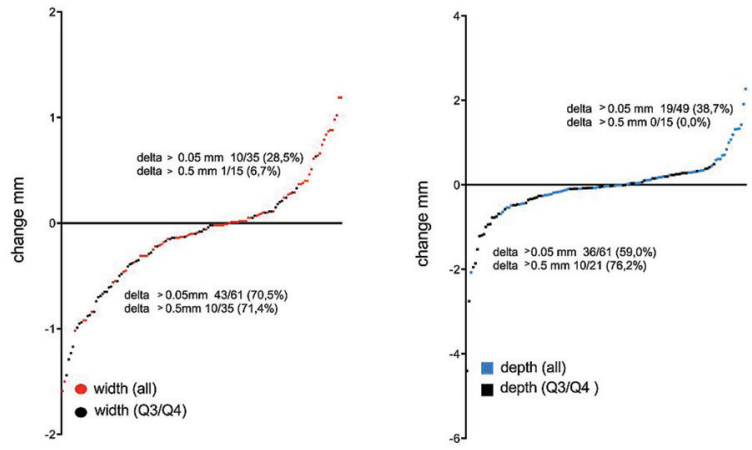

Figure 4 Size of bone erosions and evidence of repair. Mean $\pm S D$ changes of erosion width $(A)$ and erosion depth $(\mathrm{B})$ in lesions showing sclerosis at baseline and follow-up (sclerosed) and follow-up only (sclerosing). Data re shown for the quartiles of erosion width and depth at baseline ( 01 to 04 , with 01 resembling the smallest lesions and 04 the largest lesions). (C) Probability plot showing the absolute changes in erosion width (red) and depth (blue) for each single bone erosion. Negative values on the left indicate repair, whereas positive values on the right indicate progression of lesions. (D) Probability plot showing absolute changes in the width (left, red) and depth (right, blue) of all bone erosions as well as those of the quartiles 03 and 04 of erosion size (larger lesions, black).

repair. Importantly, the readers of the images were unaware of the changes in erosion width, erosion depth and sclerosis between baseline and follow-up, thus ruling out that judgement on the presence or absence of sclerosis influenced data on the changes in erosion width and depth.

Several previous studies have reported that repair of bone erosions can occur in RA patients. ${ }^{6-11} 13$ These studies, however, have also suggested that repair is limited and confined to a subset of lesions. ${ }^{1011} 13$ RA patients treated with TNF inhibitors appear preferentially to show evidence of repair. ${ }^{9-11} 13 \mathrm{We}$ recently confirmed this notion by a $\mu$ CT study, which showed clear evidence for the limited repair of bone erosions in patients treated with TNF inhibitors compared with patients treated with methotrexate. ${ }^{13}$ Interestingly, erosion repair on treatment with TNF inhibitors is also almost exclusively confined to larger lesions with evidence of sclerosis, which is in high accordance with our new data obtained in RA patients treated with tocilizumab. Why larger erosions show a higher likelihood of repair than smaller lesions is not fully understood, but may involve contact with the bone marrow space required to induce the skeletal repair process and time factors, as osteoblastic responses require time to allow sufficient cell differentiation, deposition of matrix and mineralisation and larger lesions may be older.

Whether there is evidence of erosion repair in tocilizumabtreated RA patients, as previously reported in TNF inhibitor-treated patients, remains speculative. Despite similar baseline erosion size and a very similar mean change in the size of bone erosions after 1 year among the previously analysed TNF inhibitor-treated RA patient cohort and this cohort of tocilizumab-treated RA patients, their baseline erosion count was substantially different, with significantly more baseline bone erosions in the tocilizumab cohort. Therefore, we could not perform a direct comparison of the two patient groups as the likelihood of repair may depend on the baseline erosion count. It can be speculated, however, that the degree of erosion repair is comparable in tocilizumab and TNF inhibitor-treated RA patients as the higher baseline erosion count in the tocilizumab cohort is a negative selection bias and may underestimate the potential of tocilizumab to induce repair of existing bone lesions. Qualitative differences, however, may exist in the repair of erosions between IL- 6 and TNF blockade. IL- 6 blockade thus also affects the size of the cortical break (erosion width), whereas erosion repair during TNF blockade is exclusively based on the reduction of erosion depth, ${ }^{13}$ which may indicate the preferential use of either periosteal and endosteal repair processes among these two cytokine-blocking modalities.

The pathophysiological background of the limited repair of bone erosions in RA patients treated with tocilizumab is probably based on the effects of IL- 6 on bone remodelling. IL- 6 is a strong inducer of osteoclast differentiation by at least two different pathways. It can directly stimulate differentiation of mononuclear cells to osteoclasts by forming complexes with the soluble IL-6R followed by stimulation of osteoclasts lineage cells through the membrane-bound glycoprotein 130. ${ }^{18-21}$ Blockade of the IL-6R by neutralising antibodies has recently been shown to abrogate this direct osteoclastogenic effect of IL- $6 .^{22}$ In addition, IL- 6 also acts on osteoblast lineage cells, where it induces RANKL and indirectly stimulates osteoclastogenesis. ${ }^{23-27}$ Both the direct and indirect inhibition of osteoclast differentiation explains the strong structure-sparing effects of tocilizumab and also represents a prerequisite for allowing the repair of erosions. Repair of erosions requires the attraction and differentiation of osteoblast precursors to the sites of bone erosion. This process can only occur when osteoclast-mediated bone resorption is turned off, allowing the accessibility of eroded bone surface to osteoblasts. Inhibition of osteoclast-mediated bone resorption by tocilizumab can thus be seen as an important step towards bone repair.

However, whether toclizumab fosters new bone formation, which is compromised in bone erosions of patients with $\mathrm{RA}^{27}$ 
by, for instance, affecting osteoblast recruitment and differentiation is not fully clear. Strong evidence for a shift towards increased bone formation, however, comes from the sequential analysis of bone biomarkers in the randomised controlled OPTION trial. ${ }^{28}$ In that study, biomarkers of bone formation such as osteoclacin and N-terminal propeptide of type I collagen showed a significant increase from baseline in response to tocilizumab. Preliminary data from the effects of tocilizumab in juvenile idiopathic arthritis, in which bone formation is blunted by inflammation and leads to growth retardation of the affected child, suggests that the blockade of IL-6R restores normal bone formation facilitating normal skeletal development and repair. ${ }^{29}$ 30 These favourable effects of IL-6R blockade on bone formation may be primarily based on the effective blockade of inflammation as the role of IL- 6 on osteoblast lineage cells is not necessarily detrimental. Although data have suggested that IL- 6 may block osteoblast function, ${ }^{31}$ and mice transgenic for IL- 6 show low bone formation, ${ }^{32}$ other data revealed that IL- 6 is important for osteoblast recruitment and may even foster osteoblast differentiation by the induction of insulin-like growth factor 1. . $^{33-35}$

In summary, these data show that Il-6R blockade is associated with repair of bone erosions in patients with RA. Repair is based on reduction in the width and depth of the erosion, which is somewhat different from the effect of TNF inhibitors, in which repair is based on lowering erosion depth but not width. Repair with tocilizumab is, however, limited, as observed with TNF inhibitors, and does not lead to complete healing of erosions, which suggests that current antirheumatic therapies have a rather limited potential to induce the repair of bone lesions.

Contributors SF, JR, SS and KE performed data acquisition and analysis. ME performed statistical analysis and GS drafted the manuscript.

Funding This study was supported by the Deutsche Forschungsgemeinschaft (FG 661/TP4 and SPP1468-IMMUNOBONE), the Bundesministerium für Bildung und Forschung (BMBF; project ANCYLOSS), the MASTERSWITCH project of the European Union and the IMI funded project BT Cure.

Competing interests None.

Ethics approval Approval from the University of Erlangen-Nuremberg ethics committee and national radiation safety agency (Bundesamt für Strahlenschutz) was obtained for the study.

Patient consent Obtained.

Provenance and peer review Not commissioned; externally peer reviewed.

\section{REFERENCES}

1. van der Heijde DM. Joint erosions and patients with early rheumatoid arthritis. Br J Rheumatol 1995;34 (Suppl. 2):74-8.

2. Welsing PM, van Gestel AM, Swinkels HL, et al. The relationship between disease activity, joint destruction, and functional capacity over the course of rheumatoid arthritis. Arthritis Rheum 2001;44:2009-17.

3. Aletaha D, Smolen J, Ward MM. Measuring function in rheumatoid arthritis: Identifying reversible and irreversible components. Arthritis Rheum 2006;54:2784-92.

4. McInnes IB, Schett G. Cytokines in the pathogenesis of rheumatoid arthritis. Nat Rev Immunol 2007:7:429-42.

5. Schett G, Stach C, Zwerina J, et al. How antirheumatic drugs protect joints from damage in rheumatoid arthritis. Arthritis Rheum 2008;58:2936-48.

6. van der Heijde D, Landewé R, Boonen A, et al. Expert agreement confirms that negative changes in hand and foot radiographs are a surrogate for repair in patients with rheumatoid arthritis. Arthritis Res Ther 2007;9:R62.

7. Ideguchi $\mathbf{H}, \mathrm{Ohno} \mathrm{S}$, Hattori $\mathrm{H}$, et al. Bone erosions in rheumatoid arthritis can be repaired through reduction in disease activity with conventional disease-modifying antirheumatic drugs. Arthritis Res Ther 2006;8:R76.

8. van der Linden MP, Boja R, Klarenbeek NB, et al. Repair of joint erosions in rheumatoid arthritis: prevalence and patient characteristics in a large inception cohort. Ann Rheum Dis 2010;69:727-9.

9. Lukas C, van der Heijde D, Fatenajad S, et al. Repair of erosions occurs almost exclusively in damaged joints without swelling. Ann Rheum Dis 2010;69:851-5.

10. Møller Døhn U, Boonen A, Hetland ML, et al. Erosive progression is minimal, but erosion healing rare, in patients with rheumatoid arthritis treated with adalimumab.
A 1 year investigator-initiated follow-up study using high-resolution computed tomography as the primary outcome measure. Ann Rheum Dis 2009;68:1585-90.

11. Døhn UM, Ejbjerg B, Boonen A, et al. No overall progression and occasional repair of erosions despite persistent inflammation in adalimumab-treated rheumatoid arthritis patients: results from a longitudinal comparative MRI, ultrasonography, CT and radiography study. Ann Rheum Dis 2011;70:252-8.

12. Stach CM, Bäuerle M, Englbrecht $M$, et al. Periarticular bone structure in rheumatoid arthritis patients and healthy individuals assessed by high-resolution computed tomography. Arthritis Rheum 2010;62:330-9.

13. Finzel S, Rech J, Schmidt S, et al. Repair of bone erosions in rheumatoid arthritis treated with tumour necrosis factor inhibitors is based on bone apposition at the base of the erosion. Ann Rheum Dis 2011;70:1587-93.

14. Nishimoto N, Kishimoto T. Interleukin 6: from bench to bedside. Nat Clin Pract Rheumatol 2006;2:619-26.

15. Arnett FC, Edworthy SM, Bloch DA, et al. The American Rheumatism Association 1987 revised criteria for the classification of rheumatoid arthritis. Arthritis Rheum 1988;31:315-24.

16. Aletaha D, Neogi T, Silman AJ, et al. 2010 Rheumatoid arthritis classification criteria: an American College of Rheumatology/European League Against Rheumatism collaborative initiative. Ann Rheum Dis 2010;69:1580-8.

17. Bruynesteyn K, Boers M, Kostense P, et al. Deciding on progression of joint damage in paired films of individual patients: smallest detectable difference or change. Ann Rheum Dis 2005;64:179-82.

18. Tamura T, Udagawa N, Takahashi N, et al. Soluble interleukin-6 receptor triggers osteoclast formation by interleukin 6. Proc Natl Acad Sci U S A 1993;90:11924-8.

19. Kotake S, Sato K, Kim KJ, et al. Interleukin-6 and soluble interleukin-6 receptors in the synovial fluids from rheumatoid arthritis patients are responsible for osteoclast-like cell formation. J Bone Miner Res 1996;11:88-95.

20. Udagawa N, Takahashi N, Katagiri T, et al. Interleukin (IL)-6 induction of osteoclast differentiation depends on IL-6 receptors expressed on osteoblastic cells but not on osteoclast progenitors. J Exp Med 1995;182:1461-8.

21. Liu XH, Kirschenbaum A, Yao S, et al. Cross-talk between the interleukin-6 and prostaglandin $\mathrm{E}(2)$ signaling systems results in enhancement of osteoclastogenesis through effects on the osteoprotegerin/receptor activator of nuclear factor- $\kappa B$ (RANK) ligand/RANK system. Endocrinology 2005;146:1991-8.

22. Axmann R, Böhm C, Krönke G, et al. Inhibition of interleukin-6 receptor directly blocks osteoclast formation in vitro and in vivo. Arthritis Rheum 2009;60:2747-56.

23. Palmqvist $\mathbf{P}$, Persson E, Conaway HH, et al. IL-6, leukemia inhibitory factor, and oncostatin $\mathrm{M}$ stimulate bone resorption and regulate the expression of receptor activator of NF-kappa B ligand, osteoprotegerin, and receptor activator of NF- $\mathrm{KB}$ in mouse calvariae. J Immunol 2002;169:3353-62.

24. Wong PK, Quinn JM, Sims NA, et al. Interleukin-6 modulates production of T lymphocyte-derived cytokines in antigen-induced arthritis and drives inflammationinduced osteoclastogenesis. Arthritis Rheum 2006;54:158-68.

25. Kudo 0, Sabokbar A, Pocock A, et al. Interleukin-6 and interleukin-11 support human osteoclast formation by a RANKL-independent mechanism. Bone 2003;32:1-7.

26. Ohsaki Y, Takahashi S, Scarcez T, et al. Evidence for an autocrine/paracrine role for interleukin-6 in bone resorption by giant cells from giant cell tumors of bone. Endocrinology 1992;131:2229-34.

27. Walsh NC, Reinwald S, Manning CA, et al. Osteoblast function is compromised at sites of focal bone erosion in inflammatory arthritis. J Bone Miner Res 2009:24:1572-85

28. Garnero $\mathbf{P}$, Thompson E, Woodworth T, et al. Rapid and sustained improvement in bone and cartilage turnover markers with the anti-interleukin-6 receptor inhibitor tocilizumab plus methotrexate in rheumatoid arthritis patients with an inadequate response to methotrexate: results from a substudy of the multicenter double-blind, placebo-controlled trial of tocilizumab in inadequate responders to methotrexate alone. Arthritis Rheum 2010;62:33-43.

29. Inaba $\mathbf{Y}$, Ozawa R, Imagawa $T$, et al. Radiographic improvement of damaged large joints in children with systemic juvenile idiopathic arthritis following tocilizumab treatment. Ann Rheum Dis 2011;70:1693-5.

30. De Benedetti F, Rucci N, Del Fattore A, et al. Impaired skeletal development in interleukin-6-transgenic mice: a model for the impact of chronic inflammation on the growing skeletal system. Arthritis Rheum 2006;54:3551-63.

31. Hughes FJ, Howells GL. Interleukin-6 inhibits bone formation in vitro. Bone Miner 1993;21:21-8.

32. Kitamura H, Kawata $\mathrm{H}$, Takahashi F, et al. Bone marrow neutrophilia and suppressed bone turnover in human interleukin-6 transgenic mice. A cellular relationship among hematopoietic cells, osteoblasts, and osteoclasts mediated by stromal cells in bone marrow. Am J Pathol 1995;147:1682-92.

33. Franchimont $\mathbf{N}$, Gangji V, Durant D, et al. Interleukin-6 with its soluble receptor enhances the expression of insulin-like growth factor-l in osteoblasts. Endocrinology 1997; 138:5248-55

34. Karadag A, Scutt AM, Croucher PI. Human myeloma cells promote the recruitment of osteoblast precursors: mediation by interleukin- 6 and soluble interleukin-6 receptor J Bone Miner Res 2000:15:1935-43.

35. Franchimont $\mathbf{N}$, Wertz S, Malaise M. Interleukin-6: an osteotropic factor influencing bone formation? Bone 2005; 37:601-6. 


\section{A RD Interleukin-6 receptor blockade induces limited repair of bone erosions in rheumatoid arthritis: a micro CT study}

Stephanie Finzel, Juergen Rech, Sarah Schmidt, Klaus Engelke, Matthias Englbrecht and Georg Schett

Ann Rheum Dis 2013 72: 396-400 originally published online May 14, 2012

doi: 10.1136/annrheumdis-2011-201075

Updated information and services can be found at:

http://ard.bmj.com/content/72/3/396

\section{These include:}

References This article cites 35 articles, 4 of which you can access for free at: http://ard.bmj.com/content/72/3/396\#BIBL

\section{Email alerting} service

Receive free email alerts when new articles cite this article. Sign up in the box at the top right corner of the online article.

Topic

Articles on similar topics can be found in the following collections Collections

\section{Notes}

To request permissions go to:

http://group.bmj.com/group/rights-licensing/permissions

To order reprints go to:

http://journals.bmj.com/cgi/reprintform

To subscribe to BMJ go to:

http://group.bmj.com/subscribe/ 